\title{
On the Performance of the Predicted Energy Efficient Bee-Inspired Routing (PEEBR)
}

\author{
Imane M. A. Fahmy \\ Computer \& Information Sciences \\ department, Institute of Statistical \\ Studies and Research, Cairo \\ University, Giza, Egypt
}

\author{
Laila Nassef \\ Faculty of Computing and \\ Information Technology, King \\ Abdulaziz University, Jeddah, \\ Kingdom of Saudi Arabia
}

\author{
Hesham A. Hefny \\ Computer \& Information Sciences \\ department, Institute of Statistical \\ Studies and Research, Cairo \\ University, Giza, Egypt
}

\begin{abstract}
The Predictive Energy Efficient Bee Routing PEEBR is a swarm intelligent reactive routing algorithm inspired from the bees food search behavior. PEEBR aims to optimize path selection in the Mobile Ad-hoc Network MANET based on energy consumption prediction. It uses Artificial Bees Colony ABC Optimization model and two types of bee agents: The scout bee for exploration phase and the forager bee for evaluation and exploitation phases. PEEBR considers the predicted mobile nodes battery residual power and the expected energy consumption for packet reception and relaying of these nodes along each of the potential routing paths between a source and destination pair. In this research paper, the performance of the proposed and improved PEEBR algorithm is evaluated in terms of energy consumption efficiency and throughput compared to two state-ofart ad-hoc routing protocols: The Ad-hoc On-demand Distance Vector AODV and the Destination Sequenced Distance Vector DSDV for various MANET sizes.
\end{abstract}

Keywords-PEEBR; Reactive protocol; path selection; MANET; ABC; energy consumption; battery residual power; $A O D V ; D S D V$

\section{INTRODUCTION}

The Mobile Ad-hoc Networks MANETs require competent routing protocols since they need to maintain a satisfactory performance as their nodes dynamically move and transmission properties change. Every node in MANETs should achieve two fundamental functions: It acts primarily as a transmitting or receiving point, and as a routing point to relay communicated packets destined for other nodes. Due to the limited communication range of wireless interface, a data packet has to be transferred via several intermediate nodes (Multi-hop routing). Moreover, MANET nodes have limited rechargeable battery power. Thus, the routing mechanism is the most critical and challenging problem in MANETs. In order to solve the routing problem without draining the MANET nodes batteries, a group of MANET energy efficient or power aware routing protocols have emerged as in [1-7].

Swarm Intelligence SI is a computational intelligence approach, as described by [8] that is based on the study of collective behavior of social insects in decentralized, selforganized systems. Ant Colony Optimization introduced by [9] and Bee Colony Optimization by [10] are widely studied among the other Swarm Intelligence techniques applied for networks. Swarm Intelligence SI is a computational intelligence approach, as described by [11]. SI involves a collective behavior of autonomous agents that locally interact with each other in a distributed environment to solve a given problem in the hope of finding a global solution to the problem as defined by [12]. These new SI optimization models have attracted the attention of researchers because they are more robust, reliable, and scalable than other conventional routing algorithms. Since they do not involve more control packets to maintain paths when network topology changes, they are suitable for mobile ad-hoc networks where nodes move dynamically and topology changes frequently. These natureinspired routing protocols considered the limited resources and highly dynamic environment, as well as the restriction on the exchange of routing information.

Artificial Bee Colony ABC Optimization model proposed in [13] and [14] is a new paradigm of SI that mainly requires two types of agents for routing: scouts, who discover ondemand new routes (paths) to the destinations and foragers, who transport data packets and simultaneously evaluate the quality of the discovered routes based on energy amount expected to be consumed along the path and the nodes batteries residual power. The foragers sense the state of the network, utilize measured metrics to rate different routes in MANET, and then choose the appropriate optimal path for routing of data packets with the aim of maximizing network lifetime.

The Predictive Energy Efficient Bee Routing PEEBR introduced in [16] is a reactive MANET routing algorithm inspired from the natural bees food search behavior. PEEBR's routing technique tends to determine the optimal routing path based on its goodness ratio. The path goodness ratio is a combination of two energetic parameters: the expected energy consumption and the nodes batteries residual power for each potential path.

The paper is organized as follows: The second section presents briefly the routing protocols classification. Some related research works are discussed by the third section. The improved Predictive Energy Efficient Bee Routing (PEEBR) algorithm is described in the fourth section. The improved PEEBR's algorithm simulation results are shown and analyzed in the fifth section. Finally, the sixth section concludes the paper's research goal and future research work.

\section{Routing PRotocols ClassificAtion}

In Mobile Ad-hoc Networks MANETs, there are different categories of routing protocols. For unicast routing protocols, 
there are four main types of routing protocols according to the routing mechanism employed to discover, control, maintain, memorize or update the path between a specified source and destination nodes in MANET. The proactive routing depends on a routing table stored and regularly updated at each mobile node. While the reactive routing tends to discover a sourcedestination path on-demand whenever requested. A hybrid routing protocol benefits from both proactive and reactive to make a more reliable and scalable routing by dividing the MANET area into overlapping zones or clusters communicating proactively locally (within the same zone) and reactively to reach a destination in different zone. Finally, in hierarchical routing, each node has a hierarchical ID, which is a sequence of the MAC addresses from the top hierarchy to the source node [15].

According to figure 1, the Destination Sequenced Distance Vector DSDV is a distance vector proactive routing protocol. On the other hand, the Ad-hoc On-demand Distance Vector AODV and the newly proposed Predictive Energy Efficient Bee Routing PEEBR are considered reactive on-demand routing protocols. Finally, the Zone-based Routing Protocol $\mathrm{ZRP}$ is a hybrid routing protocol.

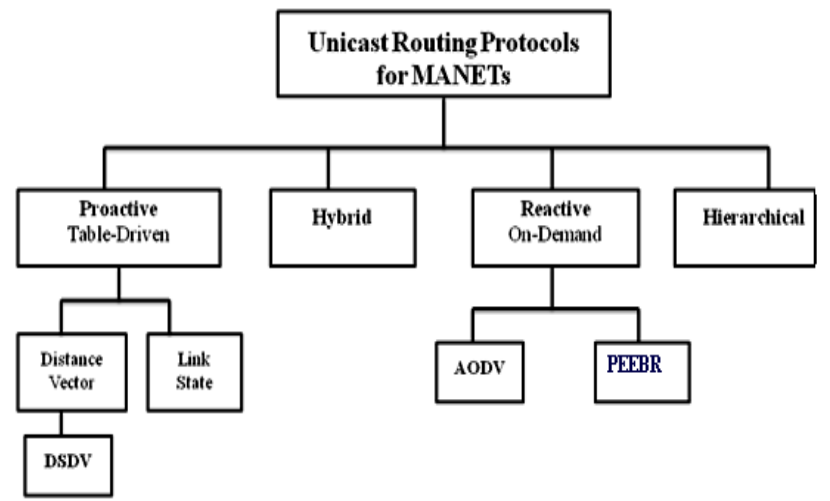

Fig. 1. Unicast MANET Routing Protocols Classification

While AODV, DSDV and ZRP are considered the state-ofart routing protocols for MANETs from the literature, PEEBR and BeeAdHoc [17] could be considered Bio/Nature inspired routing protocols. Among their common features are: multi paths discovery and probabilistic distribution of data traffic on these multi paths to achieve better performance.

The proposed Predictive Energy Efficient Bee-inspired Routing protocol PEEBR by [16] was inspired from the honey bees food search process. Particularly, the two essential groups of bees involved in food source discovery are: The scouts and the foragers. PEEBR inspired by the ABC model is an algorithm for path selection optimization based on energy prediction and consumption efficiency as well as mobile nodes battery residual power maximization in MANETs in order to increase the network lifetime.

\section{RELATED WORK}

Recently, some research works have emerged for solving the MANET's routing problem and are inspired from the natural bee's behavior as discussed in the following subsections.

\section{A. BeeHive Routing Protocol}

Wedde, Farooq, and Zhang in [19] introduced a novel routing algorithm called "BeeHive" inspired by the communicative and evaluative methods and procedures of honey bees. In this algorithm, bee agents travel through network regions called foraging zones. On their way, their information on the network state is delivered for updating the local routing tables. BeeHive was fault tolerant, scalable, and relies completely on local, or regional, information, respectively. They have also demonstrated through extensive simulations that the reactive BeeHive routing protocol achieves a similar or better performance compared to state-ofthe-art Mobile Ad-hoc Networks routing algorithms such as: AODV, DSDV and DSR.

In BeeHive algorithm, the bee's colony architecture consists of three main exploitation floors as described below:

1) The entrance floor: At this floor the scouts come back to the hive (from their exploration phase). This is the interface to lower level (MAC layer).

2) The dance floor: This is the floor where the dance takes place. The foragers update the routing information of hive's bees (node).

3) The packing floor: This floor is where the worker bees come back with honey to be packed (path control information to update tables). It is responsible of interacting with higher level layer (transport layer).

\section{B. BeeAdHoc Routing Protocol}

H. F. Wedde et al. in [17], then presented a new routing algorithm for MANET which is also inspired by the honey bee behavior called BeeAdHoc. The algorithm is simple and mainly needs two types of messages for routing: the scouts: They discover on-demand (reactive) new routes to the destinations. Then, the forgers: which transport data packets and simultaneously evaluate the quality of the discovered routes. The BeeAdHoc routing as shown by figure 2 [17] considers each node in the network as a hive. Each node periodically sends out bee agents: Scouts to explore the network and collect information about any available food sources regardless of their quality. The exploration process achieved by the scout bees could be described and mapped onto the following steps in MANET: Scouts are broadcasted. A TTL (Time To Live packet) is set for each Scout. Then, Scouts take a backward journey to the source (hive) on the same route. At last, Scouts recruit foragers when they are back to the hive by dancing to guide them to the food direction (angle) from the hive.

BeeAdHoc protocol considers the dance floor as the routing table where the bee agents provide the information about the quality of the path they have traversed. Then the exploitation process will be achieved by the foragers and the main workers. Foragers receive data packets from the transport layer (provided by the scouts) and after determining the path quality, they deliver it also by dance to the main workers. Finally, the main workers who receive packets from the transport layer (foragers) are recruited by the foragers such that every worker has a food source. 


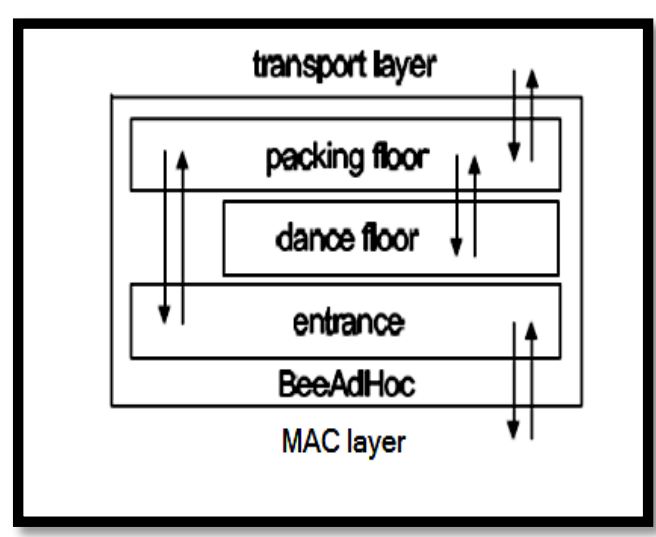

Fig. 2. BeeAdHoc algorithm architecture [17]

It is noteworthy to mention that Beehive and BeeAdHoc routing protocols have not utilized the $\mathrm{ABC}$ optimization model in their mechanism or network parameters optimization.

\section{The IMPRoved PREDICTIVE ENERGY EFFICIENT BEE- INSPIRED ROUTING (PEEBR)}

In bees search process, there are three main phases: First, the scouts seek out all potential food sources which is equivalent to finding all potential MANET routing paths. Then, the foragers assign each discovered food source (routing path) a certain probability according to its quality (nectar amount) interpreted as the link cost for MANET. Finally, the worker bees collect the nectar from the food source with the highest quality according to the qualification probability assigned by the foragers which is equivalent to the optimal path selection according to its quality to communicate the data stream of packets on it in MANETs.

The optimal path selection is based on two main parameters: The average energy consumed by all nodes along each potential path and the nodes average battery residual power together with the hop count. These parameters reflect the path goodness assigned by forager bee agent. The path with the highest goodness ratio should be considered as the optimal path. In PEEBR, the optimal path discovery process from source $n_{s}$ to the destination node $n_{d}$ could be described as follows:

\section{A. The Scout Bee}

Source node $\mathrm{n}_{\mathrm{s}}$, in order to route efficiently its packets to a destination node $\mathrm{n}_{\mathrm{d}}$, floods a "Scout packet" associated with a TTL (Time-To-Live) to all $\mathrm{j}$ neighboring nodes. For each "Scout cycle", each "Scout" flies over one of the $\mathrm{j}$ potential routes $R_{j}$ until it reaches destination node $n_{d}$.

If the TTL packet expires, the "Scout" bee agent packet will die indicating failure to reach destination to the source and the corresponding routing path will be avoided.

When a bee agent reaches the destination node $n_{d}$, it is sent back to its source $n_{s}$ through the same traveled route. The backward packet from destination node $\mathrm{n}_{\mathrm{d}}$ to source node $\mathrm{n}_{\mathrm{s}}$, "Scout packet", collects the potential route's routing information. It counts number of hops $h\left(R_{j}\right)$.
Then, it collects each route nodes residual battery power $B\left({ }_{n j i}\right)$ where $i=1$ to $N_{j}$ nodes and $j=1$ to $M$ paths. Finally, it memorizes the amount of receiving power consumed.

\section{B. The Forager Bee}

At the source node $\mathrm{n}_{\mathrm{s}}$, the "forager" evaluation process starts by calculating the predicted amount of energy to be consumed for each "Backward Scout" discovered route. Each potential route cost $f\left(R_{j}\right)$ is calculated for each route $\mathrm{Rj}$ dependent on its hop count $h\left(R_{j}\right)$, its nodes residual battery power $B\left({ }_{n j i}\right)$ and its expected amount of receiving power consumed using expression (6).

The "Forager" associates a fitness value fit $\left(R_{j}\right)$ and a goodness ratio of each route $G\left(R_{j}\right)$ as deduced from expressions (7) and (8). At the end of each foraging iteration, each potential path nodes battery residual power $B\left(n_{i j}\right)$ should be decayed exponentially as computed by (9) to reflect the real world's energy consumption.

Therefore, the optimal route $R_{o}$ between $n_{s}$ and $n_{d}$ is the route with the maximum goodness ratio as given by expression (10).

The other potential routing paths are memorized by source node $\mathrm{n}_{\mathrm{s}}$ (for a time interval in communication) in order to be used if any failure occurred during transmission on the optimal route $\mathrm{R}_{\mathrm{o}}$ but with respect to their goodness ratio. Finally, a new "Scout cycle" is launched until the maximum number of iterations is reached or a minimal fitness value.

A fault-tolerant and efficient routing protocol is the one that encounters the energy consumption among the other routing information collected before choosing a path and starting transmission. The Artificial Bees Colony ABC model is used by this research in order to employ artificial bee agents that travel from the source node to the destination. The bee agents travel on all potential paths, collect energy information about all the nodes along the path, predict the amount of energy that will be consumed while routing and choose the optimal path. The energy information about a path should reveal:

- Each node's battery power residual: if it is below a certain predetermined threshold, then the whole path cannot be selected to transmit the data packets

- The total energy to be consumed by the path nodes: This parameter will indicate the efficiency of the path from energetic point of view, in order to route the packets over the path that consumes less energy. The path that consumes less energy is often with the least number of hops since it will pass by the least number of nodes.

Therefore, the proposed Predictive Energy-Efficient Bee Routing (PEEBR) is assumed to be a reactive routing algorithm that enables a source node to discover the optimal path to a destination node based on the expected energy to be consumed during packets reception and the path nodes residual battery power. 
However, PEEBR algorithm could not benefit of the Artificial Bee Colony (ABC)'s food source position optimization functions for the following reasons:

- The nodes random mobility (Any recorded path will not remain the same).

- The reactive nature of the protocol that avoids an inefficient overhead that may be caused by the intention to save and update all paths to all nodes in MANET which results in an inefficient utilization of the MANET's resource: the nodes memory and power.

In table 1, the inspired ABC model's elements are mapped to the PEEBR's algorithm elements together with their optimization interpretation in order to clarify the inspired parts of the ABC model including:

- The fitness function.

- The probability associated with each potential path.

TABLE I. MAPPING ABC MODEL ONTO PEEBR AlgORITHM's OPTIMIZATION PARAMETERS

\begin{tabular}{|c|c|c|}
\hline $\mathbf{A B C}$ & PEEBR & Optimization \\
\hline Food Source Position & $\begin{array}{l}\text { Path between a } \\
\text { source node } \& \\
\text { destination }\end{array}$ & $\begin{array}{l}\text { Possible } \\
\text { solution to } \\
\text { optimize }\end{array}$ \\
\hline Amount of nectar & $\begin{array}{l}\text { Average path nodes } \\
\text { residual power }\end{array}$ & $\begin{array}{c}\text { Solution } \\
\text { quality }\end{array}$ \\
\hline Number of employed bees & $\begin{array}{c}\text { Number of potential } \\
\text { paths }\end{array}$ & $\begin{array}{c}\text { Number of } \\
\text { solutions }\end{array}$ \\
\hline $\begin{array}{l}f_{i} \\
=\frac{1}{D_{\text {Train }}} \sum_{j=1}^{D_{\text {Train }}} d\left(x_{j}, P_{i}^{C L_{\text {Known }}\left(x_{j}\right)}\right)\end{array}$ & $\begin{array}{l}\boldsymbol{f}\left(\boldsymbol{R}_{j}\right) \\
=\boldsymbol{h}\left(\boldsymbol{R}_{\boldsymbol{j}}\right) \sum_{i=1}^{\mathrm{Nj}} \frac{\boldsymbol{E}_{r}\left(\boldsymbol{n}_{i j}\right)}{\boldsymbol{B}\left(\boldsymbol{n}_{\boldsymbol{i} j}\right)}\end{array}$ & Cost Function \\
\hline$f i t_{i}=\frac{1}{1+f_{i}}$ & $\begin{array}{l}f i t\left(R_{j}\right) \\
=\frac{1}{1+f\left(R_{j}\right)}\end{array}$ & $\begin{array}{l}\text { Fitness } \\
\text { Function }\end{array}$ \\
\hline$D_{\text {Train }}$ & $\mathrm{N}_{\mathrm{j}}$ & $\begin{array}{l}\text { Number of } \\
\text { nodes along } \\
\text { route } R_{j}\end{array}$ \\
\hline SN Sources Number & M paths & $\begin{array}{c}\text { Number of } \\
\text { Solution }\end{array}$ \\
\hline$P_{i}=\frac{f i t_{i}}{\sum_{n=1}^{S N} f i t_{n}}$ & $\begin{array}{l}\mathbf{P}\left(\mathbf{R}_{\mathbf{j}}\right) \\
=\frac{\text { fit }\left(R_{j}\right)}{\sum_{j=1}^{\mathrm{M}} \text { fit }\left(R_{j}\right)}\end{array}$ & $\begin{array}{l}\text { Probability of } \\
\text { solution }\end{array}$ \\
\hline
\end{tabular}

The generic expression used to calculate $\mathrm{E}(p)$ the energy required to transmit a packet $p$ is given in equations (1) to (5) as in [20]. $\mathrm{E}(p)$ in joules (or milli-joules) is given by (1):

$$
E(p)=i * v * t_{p}
$$

Where $i$ represents the current consumption, $v$ is the voltage used and $t_{p}$ is the required time in seconds to transmit a packet given by (2):

$$
t_{p}=\left(\frac{p_{h}}{6 * 10^{6}}+\frac{p_{d}}{54 * 10^{6}}\right)
$$

Where $p_{h}$ is the packet header size and $p_{d}$ is the packet data size (both in bits). Then, the energy consumed by the node in transmit mode $E_{t}(p)$ is given by (3), while the energy consumed in reception mode $E_{r}(p)$ or in overhearing mode when the node overhears the packets exchanged within its range are given by (4):

$$
\begin{aligned}
& E_{t}(p)=280 m A * v * t_{p} \\
& E_{r}(p)=E_{o}(p)=240 m A * v * t_{p}
\end{aligned}
$$

Therefore, the total amount of energy consumed at a nod $n_{i}$ is calculated by (5):

$$
E\left(n_{i}\right)=E_{t}\left(p_{n_{i}}\right)+E_{r}\left(p_{n_{i}}\right)+E_{o}\left(p_{n_{i}}\right)
$$

On the other hand, all nodes residual power $\mathrm{B}\left(\mathrm{n}_{\mathrm{ij}}\right)$ was initiated using a random value generation in a range from 1000 to 3000 joules. PEEBR's cost function combining the hop count $h\left(R_{j}\right)$ between a given source and destination nodes pair and the average predicted energy consumption $E\left(n_{i j}\right)$ as path minimizing parameters while the average path nodes battery residual power $B\left(n_{i j}\right)$ as maximizing parameter are given by (6).

$$
f\left(R_{j}\right)=h\left(R_{j}\right) \sum_{i=1}^{N_{j}} \frac{E\left(n_{i j}\right)}{B\left(n_{i j}\right)}
$$

Where $N_{j}$ is the number of nodes on a potential path $R_{j}$ among $\mathrm{M}$ potential paths between the source and destination and the path index $j=1, . ., M$. Then, the path fitness fit $\left(R_{j}\right)$ could be computed using (7).

$$
\text { fit }\left(R_{j}\right)=\frac{1}{1+f\left(R_{j}\right)}
$$

Therefore, the path goodness $G\left(R_{j}\right)$ could be computed using (8)

$$
G\left(R_{j}\right)=\frac{f i t\left(R_{j}\right)}{\sum_{j=1}^{M} \text { fit }\left(R_{j}\right)}
$$

In order to test PEEBR's performance, it was run on $\mathrm{T}_{\max }=100$ iterations. The nodes battery residual power $\mathrm{B}\left(\mathrm{n}_{\mathrm{ij}}\right)$ was decayed to reflect the real world's as given by (9):

$$
B\left(n_{i j}\right)=B\left(n_{i j}\right)^{0} * e^{\frac{-t}{\tau}}
$$

Where $\mathrm{B}\left(\mathrm{n}_{\mathrm{ij}}\right)^{0}$ is the initial node battery residual power, $\mathbf{t}$ is the iteration number and $\boldsymbol{\tau}$ is a time constant. Finally, PEEBR termination conditions were: reaching the maximum number of iterations $\mathrm{T}_{\max }$ or a minimal predefined fitness value.

The resulting optimal path $\mathbf{R}_{\mathbf{0}}$ is the path with the highest goodness ratio that is given by (10):

$$
\mathrm{R}_{\mathrm{o}}=\arg \max _{\mathrm{j}}\left\{\mathrm{G}\left(\mathrm{R}_{\mathrm{j}}\right)\right\}
$$




\section{Simulation AND Results}

In order to evaluate the proposed Predictive Energy Efficient Bee-inspired Routing PEEBR, a self-made simulator using Visual $\mathrm{C}++$ was used to simulate its performance. Since the MANET's critical resource to be efficiently consumed and saved while routing is the nodes battery power, PEEBR's key parameters are: The average energy consumption and the routing path nodes batteries residual power.

In figure 3 , it is clearly depicted the impact of increasing the number of nodes in MANET on the the average energy consumed in milli-joules. The proactive DSDV, the reactive AODV and PEEBR protocols showed similar and competitive average energy consumed at smaller MANET sizes as 10 and 20 nodes, then at 30 nodes, their consumption increases more than PEEBR which demonstrates its stability and efficiency. On the other hand, the hybrid ZRP protocol started consuming much less average energy consumption at 10 nodes, then similar to other protocols at 20 nodes, but increased by double at 30 nodes.

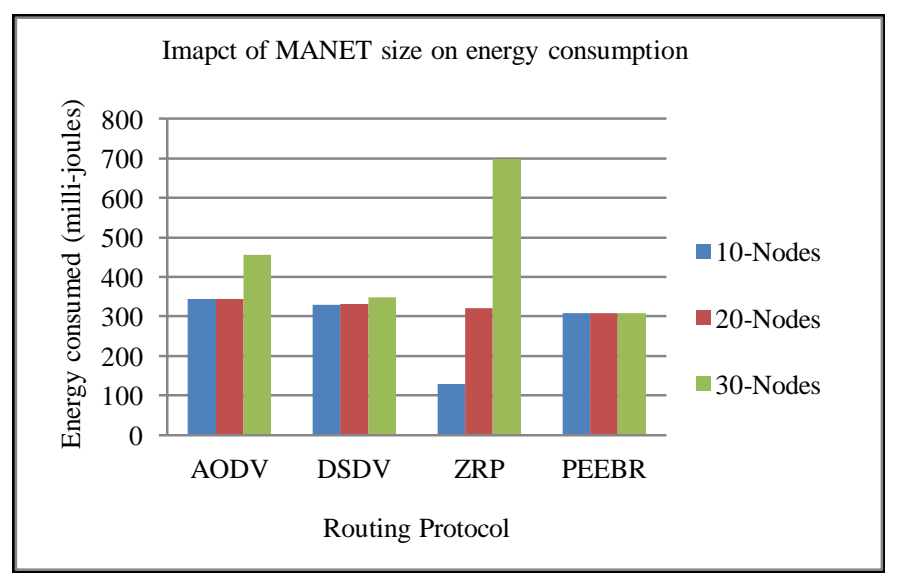

Fig. 3. The impact of varying MANET size on energy consumption by stateof-art routing protocols Vs PEEBR

On the other hand, PEEBR's performance and energy consumption efficiency was compared to another recent beeinspired routing protocol: BeeAdHoc [17]. Figure 4 shows the impact of increasing the MANET's number of nodes on the energy consumed in transporting one kilobyte of data to its destination which includes the energy consumed for both data and control traffic as defined by [18]. At 10 nodes, PEEBR consumed less energy than BeeAdHoc. Then, at 25 nodes, PEEBR's energy consumption is slightly higher by 0.09 $\mathrm{mJ} / \mathrm{KB}$ than BeeAdHoc.

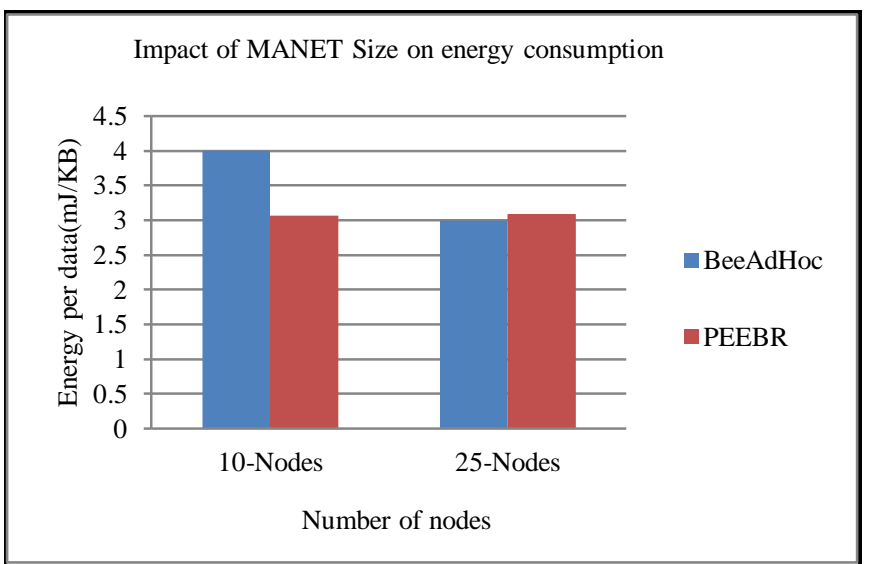

Fig. 4. The impact of varying MANET size on energy consumption by BeeAdHoc Vs PEEBR

\section{CONCLUSION AND FUTURE WORKS}

In this paper, the reactive Predictive Energy Efficient Beeinspired Routing PEEBR previously proposed in [16] was improved and its path selection optimization algorithm was described. Then, in order to evaluate PEEBR's average energy consumption efficiency in MANETs, we compared its performance to some state-of-art routing protocols as the reactive AODV, the proactive DSDV and the hybrid ZRP. Finally, PEEBR's energy consumed per data measured in $\mathrm{mJ} / \mathrm{KB}$ was compared to another bee-inspired routing protocol: BeeAdHoc. The simulation results have shown that PEEBR is a competitive energy efficient routing algorithm.

The future work for this research include evaluating PEEBR's performance for other MANET parameters comprising: Packet Delivery Ratio PDR and end-to-end delay under MANET size scenario and mobility scenario.

\section{REFERENCES}

[1] L. M. Feeney. "An energy consumption model for performance analysis of routing protocols for mobile ad hoc networks". Mobile Networks and Applications, 6(3):239-249, 2001.

[2] L.M. Feeney and M. Nilsson. "Investigating the energy consumption of a wireless network interface in an ad hoc networking environment". In Proceedings of IEEE INFOCOM, 2001.

[3] N. Nie and C. Comaniciu, "Energy efficient aodv routing in cdma ad hoc networks using beam forming" EURASIP J. Wireless Communication Networks., vol. 2006, no. 2, pp. 14-14, 2006.

[4] R. Shah and J. Rabaey, "Energy aware routing for low energy ad hoc sensor networks", Wireless Communications and Networking Conference, WCNC2002. IEEE, vol. 1, pp. 350-355 vol.1, 2002.

[5] C. E. Jones, K. M. Sivalingam, P. Agrawal, and J. -C. Chen. "A survey of energy efficient network protocols for wireless networks." Wireless Networks, 7(4):343-358, 2001. 
[6] K. Pappa, A. Athanasopoulos, E. Topalis, and S. Koubias, "Implementation of power aware features in aodv for ad hoc sensor networks a simulation study". IEEE Conference on Emerging Technologies and Factory Automation ETFA, pp.1372-1375, Sept. 2007.

[7] Cui Y., Xue Y., Nahrstedt K., “A Utility-Based Distributed Maximum Lifetime Routing Algorithm for Wireless Networks". Vehicular Technology, IEEE Transactions on vehicular technology, 55(3) (2006) 797, 2006.

[8] Kennedy J, Eberhart R. Particle swarm optimization, In Proceeding of IEEE international conference neural networks, vol. 4; pp. 1942-7, 1995.

[9] M. Dorigo, M. Birattari and Thomas Stutzle. "Ant Colony Optimization: Artificial Ants as computational intelligence technique ». Université libre de Bruxelles, Belgique IEEE Computational Intelligence magazine, November 2006

[10] D. Karaboga and B. Basturk. "A powerful and efficient algorithm for numerical function optimization: artificial bee colony (ABC) algorithm”. Springer Science+Business Media B.V., 2007

[11] Mayur Tokekar and Radhika D. Joshi, "Enhancement of Optimized Linked state routing protocol for energy conservation", CS \& IT-CSCP, 2011

[12] J. Wang, E. Osagie, P. Thulasiraman, R. K. Thulasiram, "HOPNET: A Hybrid ant colony OPtimization routing algorithm for Mobile ad hoc NETwork", Elsevier Ad Hoc Networks, June 2008.

[13] D. Karaboga and Ozturk, "A novel clustering approach: Artificial Bee Colony (ABC) algorithm”, Elsevier, Applied Soft Computing 11 (2011) 652-657, 2011
[14] D. Karaboga and B. Basturk, "On the performance of artificial bee colony (ABC) algorithm”, Elsevier, Applied Soft Computing 8 (2008) 687-697, 2008

[15] Mehran Abolhasan, Tadeusz Wysocki, Eryk Dutkiewicz, “A review of routing protocols for mobile ad hoc networks", ElSevier Computer Science, Ad Hoc Networks 2 (2004) 1-22

[16] Imane M. A. Fahmy, Laila Nassef and Hesham A. Hefny, "PEEBR: Predictive Energy Efficient Bee Routing Algorithm for Ad-hoc Wireless Mobile Networks", IEEE INFOrmatics and Systems (INFOS2012), 2012

[17] H. F. Wedde, M. Farooq, T. Pannenbaecker, B. Vogel, C. Mueller, J. Meth, and R. Jeruschkat. "BeeAdHoc: an energy efficient routing algorithm for mobile ad-hoc networks inspired by bee behavior." In Proceedings of ACM GECCO, pages 153-160, 2005

[18] Nauman Mazhar, Muddassar Farooq, "Vulnerability Analysis and Security Framework (BeeSec) for Nature Inspired MANET Routing Protocols", GECCO'07, July 7-11, 2007, London, England, United Kingdom, 2007 ACM 978-1-59593-697-4/07/0007

[19] H. F. Wedde, M. Farooq, and Y. Zhang. "Beehive: An efficient faulttolerant routing algorithm inspired by honey bee behavior". In Proceedings of ANTS Workshop, LNCS 3172, pp. 83-94. Springer Verlag, 2004.

[20] Marco Fotino, Antonio Gozzi, Floriano De Rango, Salvatore Marano, juan-Carlos Cano, Carlos Calafate, Pietro Manzoni, «Evaluating Energy-aware Behavior of Proactive and Reactive Routing Protocols for Mobile Ad Hoc Networks" 